\title{
SOME GENERALIZATIONS FOR A THEOREM BY LANDAU
}

\author{
LAURENŢIU PANAITOPOL
}

Abstract. Let $\pi(x)$ be the number of primes not exceeding $x$. E. Landau made the following conjecture: $\pi(2 x) \leqslant 2 \pi(x)$ for integer $x \geqslant 2$. In 1966 Rosser and Schoenfeld proved this conjecture. In the present paper we establish upper bounds for $\pi(x+y)$. Taking the particular case $x=y$, we find again Landau's inequality.

Mathematics subject classification (2000): 11A25, 11N05.

Key words and phrases: prime numbers, inequalities, convex function.

\section{REFERENCES}

[1] H. IshikaWA, Über die Verteilung der Primezahlen, Sci. Rep. Tokyo Univ., 2 (1934), 27-40.

2] E. Landau, Handbuch der Lehre von der Verteilung der Primzahlen, Chelsea Publ., New York, 1953.

[3] D. S. MitrinOvić, J. SÁNDOR AND B. CRSTICI, Handbook of Number Theory, Kluwer Academic Publishers, Dordrecht - Boston - London, 1996.

[4] L. PANAitopol, Several approximations of $\pi(x)$, Math. Inequal. Appl. 2 (1999), no. 3, 317-324.

[5] J. B. ROSSER AND L. SCHOENFELD, Approximate formulas for some functions of prime numbers, Illinois J. Math. 6 (1962), 64-94.

[6] J. B. Rosser AND L. SCHOENFELD, Abstract of scientific communications, Intern. Congr. Math., Moskow, (1966), Section 3, Theory of Numbers.

[7] A. SCHINZEL, Remarks on the paper "Sur certaines hypothèses concernant les nombres premiers", Acta Arith. 7 (1961), 1-8.

[8] S. L. Segal, On $\pi(x+y) \leqslant \pi(x)+\pi(y)$, Trans. Amer. Math. Soc. $104(1962)$, 523-527. 